\title{
GÉNERO, EMPLEO Y MATERNIDAD: ANÁLISIS COMPARATIVO DE MUJERES CON TRABAJO REMUNERADO Y AMAS DE CASA
}

\author{
Pilar Martínez, Ma José Carrasco, Gonzalo Aza, Isabel Espinar \\ Universidad Pontificia Comillas \\ mapi@chs.upcomillas.es
}

Ángeles Blanco

Universidad Complutense de Madrid

Recibido: 27-02-09

Aceptado: 09-03-09

\section{Resumen}

Entre los cambios sociales más destacados en las últimas décadas en España, al igual que en otros países occidentales, está el incremento espectacular del número de mujeres que, además de sus responsabilidades familiares, tienen un trabajo remunerado y lo mantienen cuando se casan y cuando tienen hijos. Este cambio ha supuesto una menor presencia de la mujer en los espacios que hasta ahora venía ocupando y ha demandado, por lo tanto, una reorganización de las estructuras y las relaciones en el hogar y en los roles que tanto varones como mujeres han venido desempeñando. Los cambios en la actividad de las mujeres se pueden rastrear a través de las tasas de empleo, el tipo de empleo, o el abandono del mercado laboral. Sin embargo, los datos relativos al cambio en los roles y en las relaciones familiares son más difíciles de documentar. En este artículo se llevará a cabo un análisis de las características del empleo en las mujeres y del contexto ideológico en que se desarrolla, atendiendo a sus implicaciones en las 
relaciones familiares. Se presentará por último un estudio empírico en el que se comparan mujeres con hijos pequeños que tienen un empleo remunerado y mujeres que no lo tienen en algunas variables personales y familiares relevantes en el tema de la relación entre familia y trabajo.

Palabras clave: Maternidad intensiva; Roles familiares tradicionales; madres empleadas; familias de doble empleo.

\begin{abstract}
The number of couples in which both partners have a job, and must balance work and family roles, has increased dramatically over the past thirty years. In Spain as in other western countries the number of women who continue in their employment when they marry and have children, has been steadily increasing. This change has brought a decrease in the woman's presence at home and a reorganization of the traditional family roles and relationships for both women and men. Although changes in women activity may be studied through their employment rate, type of employment and drop out of the labor market, it is much more difficult to study changes in family gender roles and family relationships. This article will examine women's and in particular mother's employment, taking into account the sociological patterns and the ideological context in which it occurs and its relationship with family dynamics. Finally, it will be presented an empirical research comparing mothers who are employed and mothers who are not in some relevant family and individual areas.
\end{abstract}

Key words: Intensive mothering ideology; Traditional family roles; Working mothers; dual-earner families. 


\section{Evolución del empleo de las mujeres en España}

En España el aumento del número de mujeres laboralmente activas empezó más tardíamente que en Europa, pero tras su inicio ha evolucionado de forma más acelerada, de modo que hemos pasado de un 27,1\% de mujeres activas laboralmente en 1980 al 45,79 \% en 2004. Desde entonces el ritmo de crecimiento es más estable situándose en 2007 en 49,37 (Instituto de la Mujer, 2008a). Sin embargo, hay que señalar que la tasa de población activa de mujeres en España es una de las más bajas de Europa (46\%). Con respecto a Europa, nos situamos por encima de algunos países del sur como Italia y Grecia y de algunos países del este (Polonia e Hungría), pero a gran distancia de países como Portugal, Dinamarca, Suecia o Reino Unido que oscilan entre el $50 \%$ y el $60 \%$ (Instituto de la Mujer, 2008a).

El hecho de que en España los cambios en el empleo femenino hayan sido más acelerados que en otros países de nuestro entorno, podría explicar en parte las resistencias y dificultades existentes. En una sociedad en la que durante más tiempo ha sido normal que las mujeres trabajasen exclusivamente en la esfera doméstica y los varones en la pública, se van poniendo en evidencia aquellos factores que no se adaptan a las nuevas circunstancias con la misma rapidez y que dificultan y hacen más conflictiva la actividad profesional como, por ejemplo, la escasa participación de los varones en las tareas domésticas y de crianza, la poca disponibilidad de centros de educación infantil de calidad, o la falta de adecuación de los horarios de los centros educativos a los horarios laborales. Todos estos factores que facilitan o complican el compatibilizar la vida familiar y laboral cambian más lentamente y, de hecho, España va por detrás de otros países europeos en lo que respecta a políticas que faciliten mantener el trabajo sin desatender las responsabilidades familiares. Así, por ejemplo, en el año 1998 sólo un 5\% de los niños en la franja de edad de 0 a 2 años estaban escolarizados en centros institucionalizados para cuidar niños frente a un $64 \%$ en Dinamarca o un $38 \%$ en Irlanda (VV.AA., 2004). Y con la escolarización obligatoria a 
los 6 años los problemas no se terminan ya que los horarios escolares plantean importantes dificultades a los padres hasta que sus hijos son mayores de 15 años.

Por otra parte es significativa la baja tasa de natalidad (1,27 hijos por mujer en 2002) en un país como España con una tradición cultural y unos valores muy centrados en la familia y a su vez con una baja participación femenina en el mercado de trabajo (Castles, 2003). Según algunos autores esta caída en la tasa de fertilidad en los países del sur de Europa se puede explicar desde el cambio de valores y preferencias vitales en las mujeres en las últimas décadas. La maternidad ha dejado de ser la meta prioritaria y además se percibe en conflicto con el trabajo, y las mujeres quieren compatibilizar familia y trabajo. Parecería, entonces, que esta generación de mujeres con niveles educativos más altos y que valoran mucho el trabajo remunerado, están dispuestas a hacer ajustes en su fertilidad para lograr sus objetivos profesionales. Por eso, en países como el nuestro en el que aún genera un cierto recelo el desempeño simultáneo de roles familiares y laborales ${ }^{1}$ y en el que las políticas sociales y de las empresas no facilitan esta compatibilización, las mujeres tienden a diferir la maternidad hasta que sus carreras profesionales están asentadas y a tener pocos hijos para que éstas no se vean muy alteradas (Alberdi, 1999; Esping-Andersen, 1999). Podríamos decir que España es un país en el que con frecuencia la mujer que trabaja tiene pocos hijos por la dificultad que entraña ideológica y prácticamente y las que tienen más hijos, abandonan el mercado laboral.

\footnotetext{
${ }^{1}$ Según los resultados de un trabajo de investigación del Instituto de la Mujer que encuestó a 4000 personas en edad laboral, un $45 \%$ considera que la mujer debe dejar su trabajo tras tener al primer hijo y un $63,9 \%$ de varones y un $56 \%$ de mujeres considera que las dificultades para compaginar vida familiar y laboral deterioran la educación de sus hijos/as (Instituto de la Mujer, 2005).
} 


\section{Pautas de empleo en las mujeres en relación con variables familiares}

Al analizar los perfiles de mujeres empleadas por grupos de edad se observa una pauta de compatibilización de trabajo y familia diferencial. En las mujeres mayores de 55 años es más común la pauta tradicional y secuencial de compaginar vida familiar y laboral (abandono del mercado laboral tras el matrimonio y, en mayor medida, tras el nacimiento del primer hijo y, en ocasiones, incorporación tras terminar la crianza de los hijos). Mientras tanto, en las mujeres más jóvenes (con tasas de actividad entre el $70 \%$ y $80 \%$ ) se da una pauta simultánea de compatibilización de maternidad y trabajo: no abandonan el empleo cuando tienen hijos y se mantienen en el mercado de trabajo toda la vida. (Instituto Mujer, 2008b; Montoro, 2000; Tobío, 2002). Es el grupo entre 24 y 55 años, en el que se encuentran las mujeres en edad reproductiva y con hijos pequeños, el que se ha incorporado de forma masiva al mercado laboral.

Tabla 1. Evolución de las tasas de actividad según la edad de la mujer (25-55 años)

\begin{tabular}{llll}
\hline & $\mathbf{1 9 8 7}$ & $\mathbf{1 9 9 5}$ & $\mathbf{2 0 0 7}$ \\
\hline de 25 a 29 & 60,86 & 72,31 & 81,40 \\
\hline de 30 a 34 & 50,78 & 62,79 & 79,50 \\
\hline de 35 a 39 & 40,01 & 60,33 & 74,90 \\
\hline de 40 a 44 & 35,72 & 54,51 & 72,80 \\
\hline de 45 a 49 & 33,50 & 44,40 & 68,70 \\
\hline de 50 a 54 & 27,68 & 34,88 & 59,40 \\
\hline
\end{tabular}

Fuente: Instituto de la Mujer (2008b) Las mujeres en cifras. 1983-2008. p. 78. Nota: En 2001 y 2005, el INE introduce cambios sustanciales en la metodología, por lo que los datos de 2007 no son comparables con los anteriores.

Dentro de este grupo de edad que tiene más altas tasas de empleo se observan diferencias en la tasa de actividad según el estado civil y según que tengan hijos o no. Las mujeres tienden más que en otras épocas a mantener el trabajo cuando se casan y 
tienen hijos, pero es más fácil que dejen de trabajar cuando aumenta el número de hijos y que reorganicen su actividad laboral disminuyendo el número de horas de trabajo.

Posiblemente el factor más determinante en el mantenimiento de la actividad laboral es el tener hijos y el número de hijos. A medida que éste número aumenta y, por lo tanto se incrementan los niveles de demanda de trabajo y tiempo, disminuye el número de mujeres activas laboralmente.

Gráfico 1. Tasas de empleo de varones y mujeres según tengan o no hijos menores de 12 años (20-49 años)

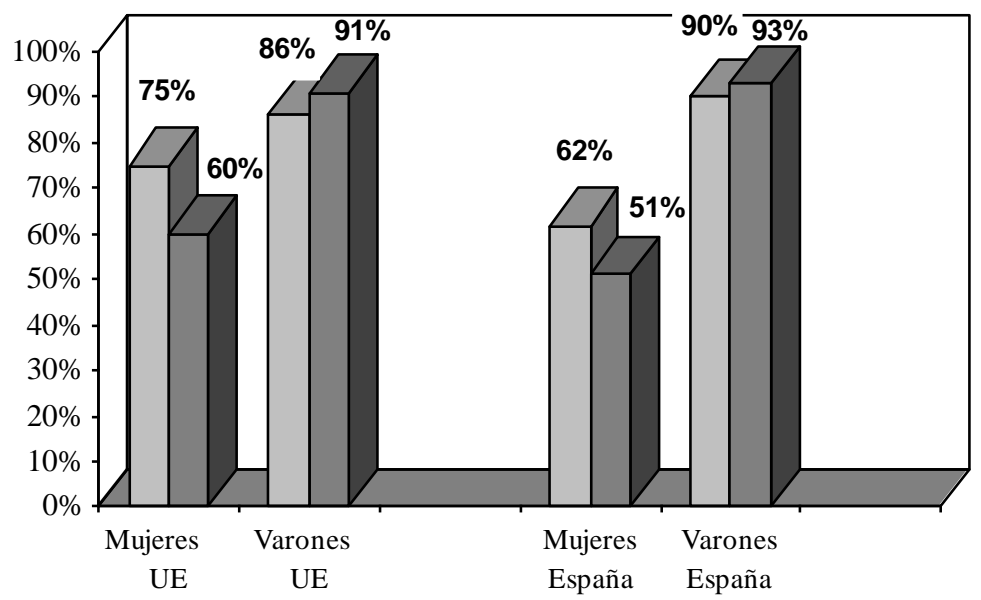

Fuente: Aliaga, C. (2005) Gender gaps in the reconciliation between work and family life.

Al tener hijos la tasa de empleo cae en 15 puntos en el conjunto de la Unión Europea y algo menos para España. Un análisis más detallado del grupo de mujeres con hijos permite observar que, con un solo hijo, disminuye relativamente poco el número de mujeres que siguen trabajando, pero a partir del nacimiento del segundo hijo y, en particular, cuando se tienen tres o más, las dificultades para armonizar exigencias laborales y familiares inciden en un descenso muy marcado de la proporción de mujeres activas laboralmente, mientras que las tasas de actividad de los varones aumentan o se mantienen en valores similares (Aliaga, 2005). Cabría, por tanto, pensar que dadas las 
dificultades para compaginar el trabajo productivo y el reproductivo muchas parejas deciden limitar el número de hijos a dos como máximo, que es la configuración familiar más frecuente en la actualidad. Pero si analizamos el fenómeno en el marco general de la Unión Europea llama la atención el que son los países con menor tasa de actividad femenina (España, Italia, Grecia) los que también tienen las tasas de natalidad más bajas, mientras en los países del Norte (Dinamarca, Suecia, Finlandia, Holanda) son compatibles las tasas de fecundidad más elevadas con una alta participación de la mujer en el mercado laboral. Por ello parece que otras variables más allá del número de hijos, explicarían los bajos niveles de actividad femenina en nuestro país.

En cuanto a los patrones más frecuentes de organización del trabajo en la pareja, se observa que analizando las familias en que conviven ambos cónyuges, en un alto porcentaje de parejas sólo el varón mantiene un trabajo remunerado, aunque este porcentaje es superior cuando tienen hijos (el $48 \%$ frente al $37 \%$ ), mientras que el perfil de los dos trabajando a tiempo completo se da en menor proporción cuando tienen hijos $(40 \%)$ y es el más frecuente cuando no los tienen $(50 \%)$.

Gráfico 2. Tipo de organización del trabajo en la pareja (20-49 años)

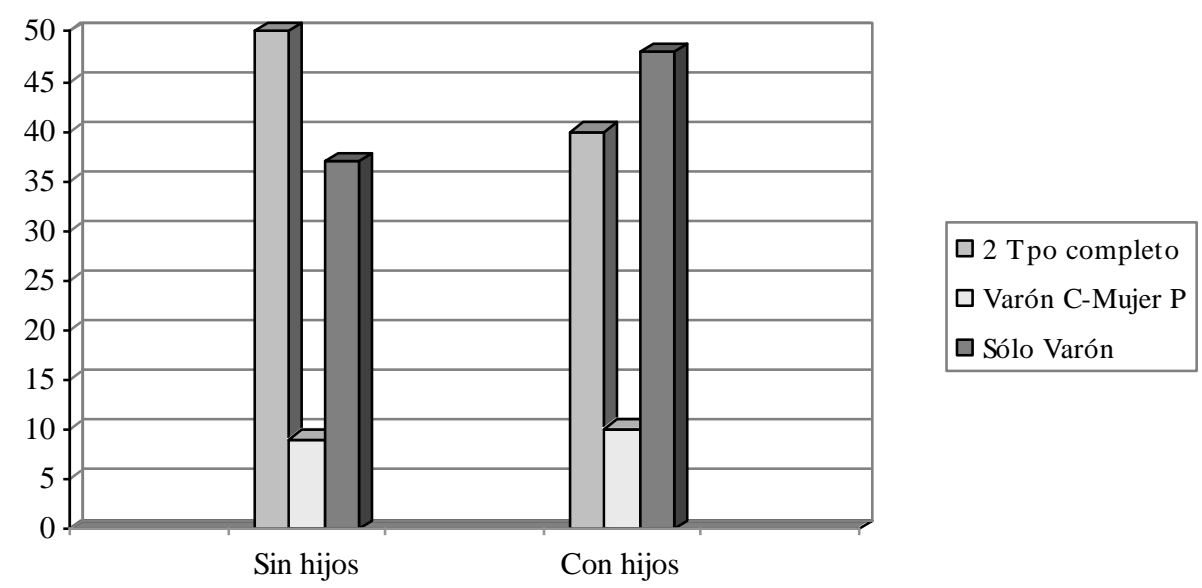

Fuente: Aliaga, C. (2005) Gender gaps in the reconciliation between work and family life. 
Si se estudian los datos existentes sobre la tasa de empleo de varones y mujeres según el número de hijos menores de 12 años, las familias se acomodan relativamente bien al nacimiento de un hijo, en lo que a mantener el trabajo remunerado de la mujer se refiere. Es a partir del nacimiento del segundo hijo y, en particular cuando se tienen tres o más, cuando las dificultades para armonizar exigencias laborales y familiares cobran mayor protagonismo apareciendo un descenso muy marcado de la proporción de mujeres activas laboralmente (Aliaga, 2005).

Si los datos existentes reflejan claramente los cambios en la ocupación y metas vitales de las mujeres, sin embargo las creencias, valores y expectativas se modifican mucho más lentamente de modo que si por un lado el tener un trabajo remunerado, desarrollarse profesionalmente y ser activa e independiente económicamente tiene una clara valoración positiva para la sociedad, a su vez, la misma sociedad y las mismas mujeres piensan que la dedicación laboral de la mujer puede ser perjudicial para su familia y para sus hijos. Estas creencias estarían relacionadas con el estereotipo de rol de madre y padre que manejan como ideal las familias y guardarían una estrecha relación con las vivencias personales y dinámicas de funcionamiento familiar.

\section{Estereotipos de rol familiar y de rol materno}

El modo en que varones y mujeres se desenvuelven en su mundo familiar y laboral y la manera de afrontar las dificultades y conflictos que puedan surgir al compatibilizar ambos roles, está estrechamente relacionado con sus creencias y vivencias en torno a qué es ser madre o padre en la sociedad actual. El estudio sobre los estereotipos y sobre los roles que se consideran adecuados en el hombre y en la mujer, permitiría determinar el nivel de interiorización de los modelos más tradicionales en un momento de cambio social que, de alguna forma, impulsa formas de funcionamiento que no se ajustan a dichos estereotipos, lo que podría tener repercusiones para buscar 
patrones de actuación más satisfactorios e incidir claramente en las experiencias de conflicto trabajo-familia, así como en los niveles de bienestar.

Los roles familiares tradicionales suelen ir asociados a un modelo de maternidad intensiva (Hays, 1996) que establece que "la maternidad es una tarea exclusiva, centrada por completo en los hijos, emocionalmente muy absorbente y que requiere una enorme inversión de tiempo. La madre desde esta ideología está dedicada al cuidado de otros: se sacrifica y no es un sujeto con sus propias necesidades e intereses" (Arendell, 2000: 1194). Las madres empleadas perciben una censura por su implicación laboral y por su ausencia en el hogar, desde las actitudes sociales enraizadas en este modelo, y deben afrontar los juicios críticos de otros y sus propios sentimientos de ambivalencia entre lograr sus mentas personales y el malestar por no ajustarse a los modelos más tradicionales de maternidad intensiva (Hays, 1998). Esta ideología transmite un mandato en el que la maternidad es parte fundamental de la identidad femenina y ser madre supone dedicarse al cuidado de los hijos, con una implicación emocional y temporal intensa, sacrificando o dejando en un segundo plano su propios deseos e intereses (Arendell, 2000). En este sistema de creencias, para un buen desarrollo de los hijos es necesaria la presencia a tiempo completo de la madre.

Por otro lado, a la vez que se plantea el posible perjuicio que genera a la familia el trabajo de la mujer, los ideales sociales proponen como modelo a seguir una mujer que trabaja y es independiente, personal y económicamente, mientras que el modelo de mujer que es ama de casa en exclusiva no está bien visto socialmente (Tobío, 2005). Una mujer dedicada en exclusiva al cuidado de la familia y a atender las demandas que se derivan de ser la responsable de gestionar y realizar las distintas tareas del hogar, se contempla como anclada en el pasado, dependiente y constreñida por un estereotipo que muestra a la mujer como alguien más débil, menos suficiente, más alejada de la actualidad social, que vive refugiada en un contexto que la aísla y distancia de una realidad que se valora como más interesante y enriquecedora personalmente. Las amas de casa son percibidas como con menos control para llevar las riendas de su vida y como alguien que vive la vida a través de los demás. Este discurso, pues, plantea un 
conflicto entre roles ya que por un lado ensalza uno de ellos como el ideal y a la vez denota al otro como degradante, lo que provoca una situación paradójica y de difícil manejo. Por ello, sería esperable que las mujeres, que tienen un trabajo remunerado, construyan su vida desde una ideología menos tradicional respecto a los papeles ideales del varón y la mujer en el ámbito familiar, mostrándose menos conformes respecto al modelo más tradicional de maternidad intensiva, que las culpabiliza y criminaliza al erigirlas, desde un ensalzamiento engañoso y mistificador, como las responsables únicas y en exclusiva del ajuste socioemocional de los hijos.

\section{Estilos educativos}

Los estilos educativos se plasman en conductas, pensamientos y sentimientos de los padres hacia sus hijos. En otras palabras, son aquellos aspectos a través de los cuales un niño aprende a modular su comportamiento de acuerdo a las demandas de la situación y a internalizar valores parentales. Diversos estudios muestran que el estilo educativo de los padres es una de las variables que más influyen en el desarrollo social, afectivo y cognitivo de los hijos (Musitu, 1995; Palacios, Hidalgo y Moreno, 2000).

En concreto, los estilos educativos hacen referencia a los modos en que los padres ejercen, por una parte, la disciplina y el control, y por otra, el afecto y apoyo con sus hijos. La combinación de estas dimensiones da lugar a estilos de educación familiar descritos en la literatura como democrático, autoritario, permisivo y negligente (MacCoby y Martin, 1983). De esta forma, en numerosos estudios se ha visto que cada uno de estos estilos tiene cierto efecto sobre el desarrollo de los hijos, de modo que un estilo autoritario, caracterizado por ser alto en control y bajo en expresión de afecto, tiende a promover en el hijo la autoexigencia, la dependencia y a tener baja autoestima. El estilo permisivo, caracterizado por utilizar pocos castigos y mostrar excesiva concesión a las demandas del hijo, fomenta la espontaneidad y creatividad, pero conlleva dificultades para controlar los impulsos y adquirir autocontrol. El estilo 
democrático, que presenta alto control y alto afecto explícito, facilita la autonomía, la autoestima y una imagen más realista de sí mismo. Por último, el estilo negligente se caracteriza por la falta de implicación afectiva con los hijos y, a la vez, ausencia de supervisión y normas que guíen su conducta y es el que más dificulta un desarrollo adecuado de los hijos.

Aunque no hay evidencia empírica que refleje diferencias entre hombres y mujeres con respecto al tipo de estilo educativo, sin embargo existen unas creencias sociales ampliamente divulgadas que plantean el deterioro que se ha producido en el contexto familiar al dedicarse ambos progenitores a desarrollar sus carreras profesionales a costa del abandono de sus funciones parentales. Esta valoración de dejación de funciones suele centrarse especialmente en la mujer trabajadora a la que se responsabiliza de emplear con mayor frecuencia estilos permisivos, debido a la vivencia de culpa, si se vive en falta por no pasar suficiente tiempo con sus hijos, y a compensar su ausencia con la utilización de una disciplina laxa e inconsistente mostrándose excesivamente tolerantes ante las demandas de los hijos.

\section{Estrés parental}

Si en la investigación empírica se ha prestado gran atención a los comportamientos, actitudes y creencias parentales que influyen en el desarrollo socioemocional de los hijos, en las últimas décadas se ha visto la necesidad de ampliar la mirada hacia los factores que influyen en los padres cuando interactúan con sus hijos (Webster-Stratton, 1990). Es decir, se hace necesario identificar qué aspectos del individuo, del contexto familiar y social y del entorno laboral inciden en que el desempeño simultáneo de roles familiares y laborales lleve asociado o no un alto nivel de tensión y estrés familiar (Martínez, 2005), que a su vez puede alterar potencialmente el funcionamiento parental y, por lo tanto, tener un impacto negativo sobre los hijos. 
De esta forma, a la expectativa social actual que recae sobre el rol paterno y materno, cada vez más exigido en su cuidado y atención a los hijos, se le añade la menor disponibilidad afectiva y de tiempo en el caso de que ambos progenitores desempeñen un trabajo remunerado. Esto puede ocasionar mayores niveles de insatisfacción y estrés, lo que puede minar la eficacia parental a la hora de criar a los hijos. En otras palabras, las vivencias y creencias sociales del modo en que hombres y mujeres entienden el ejercicio de la paternidad y maternidad pueden incrementar o disminuir la sensación de estrés y conflicto personal. Se mantiene que los miembros de la familia que experimentan estrés se vuelven más irritables y explosivos, incidiendo sobre las prácticas parentales y situando al hijo/a en riesgo de tener problemas conductuales y/o emocionales (Patterson, 1983).

En un contexto social cada vez más exigente respecto al cuidado que los padres deben proporcionar a sus hijos, las madres con un trabajo externo, que suman a las demandas del trabajo las de su familia, se sentirían sobrecargadas y desbordadas al querer responder de manera satisfactoria a los requerimientos laborales y familiares, experimentando mayores vivencias de estrés y mostrando formas de actuar que generan insatisfacción y malestar.

\section{Desarrollo socioemocional de los hijos}

Como ya hemos señalado, se constata una fuerte tendencia orientada a resaltar los desajustes que genera en los hijos el que la madre trabaje augurando graves perjuicios para la familia y la sociedad. Es frecuente escuchar en distintos foros un discurso centrado en el supuesto abandono que sufren los hijos cuyos progenitores mantienen un trabajo remunerado. Esta acusación se dirige especialmente a las mujeres que, al alterar los modelos establecidos al incorporar nuevos roles, sienten que la búsqueda de un desarrollo personal dentro del mercado laboral es percibido como desestabilizador y potencialmente peligroso para su familia. Así lo transmiten con 
frecuencia los medios de comunicación, libros de divulgación y el discurso de algunas mujeres que señala los diversos problemas que sufren aquellos niños pequeños cuyo cuidador a tiempo completo no es su madre (Tobío, 2005; Prades, 2008).

Durante años los estudios que exploraban el impacto en los hijos del trabajo materno planteaban a priori que el hecho de que madre y padre trabajaran tendría un efecto nocivo sobre los hijos (Gottfried, Gottfried y Bathurst, 1995). La investigación con respecto al efecto en los hijos del empleo materno se ha centrado en particular en niños en edad preescolar y se focaliza en la interacción padre/madre-hijo y en las conductas sociales del niño en el entorno educativo. Estas investigaciones han evaluado la cantidad y calidad de la interacción madre-hijo, el ambiente familiar y el estilo de apego entre padres e hijo. En general, los resultados indican que las madres que trabajan a tiempo completo pasan menos tiempo con sus hijos que las mujeres que trabajan a media jornada o que no trabajan. Por otro lado, las madres trabajadoras tienden a compensar su ausencia con una mayor interacción cuando están con su hijo y pasar más tiempo con él cuando están en casa y los fines de semana.

Otras investigaciones se han centrado en analizar problemas en la vinculación emocional derivados de la no exclusividad del cuidado materno, siendo este un tema particularmente controvertido, sobre todo a partir de algunas investigaciones que indicaban que los niños, que están durante largos periodos en cuidado no parental, manifiestan patrones de apego inseguro con más frecuencia que aquellos que están al cuidado exclusivo de sus madres. Se ha observado que aunque los porcentajes de apego inseguro, particularmente de tipo evitativo son más elevados, la mayor parte de los niños establecen un tipo de apego seguro con sus padres. Además, se ha informado de un aumento de conductas agresivas y de desobediencia en fases posteriores de su desarrollo en los niños que han estado en cuidado no materno desde los primeros meses de vida (Belsky, 1990). La mayor parte de los estudios no han encontrado diferencias significativas en el tipo de apego que se puedan explicar sólo por el hecho de que las madres estén empleadas, estando las diferencias encontradas más relacionadas con otro 
tipo de variables como son la sensibilidad materna a las necesidades del niño o la calidad del cuidado no materno en el que está el niño.

En el esfuerzo por aclarar estos resultados contradictorios hay que destacar en particular un estudio longitudinal muy exhaustivo que evalúa a los niños desde sus primeros meses hasta la edad escolar y en el que se analiza la influencia del cuidado no materno y las características de la familia en el estilo de apego y el desarrollo social de los niños. Este estudio tiene la ventaja de que ha sido llevado a cabo por un grupo numeroso de investigadores en el que están representadas las dos posturas a favor y en contra del cuidado no materno en los primeros años de vida (NICHD, 2003, 2005). Con respecto al estilo de apego, los resultados de esta investigación indican que la calidad de la relación madre-hijo, particularmente su sensibilidad a las necesidades del niño, influye en la seguridad del apego más que la cantidad de horas en cuidado no materno o el estatus ocupacional de la mujer.

En el análisis de las diferencias en el ajuste social de niños que han estado al cuidado de sus madres y los que han estado en cuidados no maternos, esta misma investigación señala que la diferencia más significativa tiene que ver con un nivel más alto de conductas agresivas y de desobediencia hasta los seis años en niños que no han estado a cargo de su madre en sus primeros años, particularmente si el tipo de cuidado recibido era de baja calidad y poco estable. Se observan conductas más desobedientes y agresivas, aunque las puntuaciones obtenidas mayoritariamente están en el rango de la normalidad y en muy pocos casos corresponden al extremo de riesgo clínico de presentar problemas de conducta.

\section{Análisis comparativo entre mujeres empleadas y sin empleo}

Con el objetivo de intentar aclarar algunas cuestiones relacionadas con los estereotipos de rol familiar y materno, los estilos educativos desempeñados, el estrés parental vivido y el desarrollo socioemocional de los hijos/as se recabó información de un colectivo representativo de mujeres empleadas y mujeres amas de casa en exclusiva, 
de cara a poder realizar un estudio diferencial entre mujeres con trabajo remunerado y amas de casa en algunas de las variables representativas.

Entre las Hipótesis de estudio planteadas están:

H1: Habrá diferencias entre las madres con un trabajo remunerado y las amas de casa en la valoración sobre los roles familiares y maternos, mostrando las amas de casa una ideología de rol más tradicional en ambas esferas.

H2: No habrá diferencias entre las madres con un trabajo remunerado y las amas de casa en los estilos educativos desempeñados, tanto a nivel de permisividad como de sobrerreactividad.

H3: No habrá diferencias entre las madres con un trabajo remunerado y las amas de casa en las vivencias de estrés parental.

H4: No habrá diferencias entre madres con un trabajo remunerado y amas de casa en el desarrollo socioemocional de los hijos tanto en la presencia de comportamientos agresivos como de pautas de aislamiento.

\section{Método}

\subsection{Muestra}

Este trabajo se llevó a cabo con una submuestra de una investigación más amplia sobre las relaciones familia trabajo (Martínez, Carrasco y Aza, 2007). En esta investigación se recogieron los datos de varones y mujeres con hijos entre 3 y 6 años pidiéndoles que respondieran al cuestionario con respecto a uno sólo de ellos, si tuvieran varios en esa franja de edad. Esta submuestra está compuesta por 90 mujeres, de las cuales 45 son amas de casa en exclusiva y 45 tienen un trabajo remunerado. Se han equiparado ambos grupos en aquellas variables que podrían afectar a los resultados. Así, se ha seleccionado un subgrupo equiparado al de amas de casa en nivel de ingresos, 
nivel de estudios y estructura convivencial (todas viven con su pareja). Existe una pequeña diferencia en edad (la media de las amas de casa es un año inferior a la de las mujeres con empleo) que por su escasa magnitud no se espera que pueda tener efectos relevantes sobre los resultados.

Tabla 2. Descripción de la muestra

\begin{tabular}{lcc}
\hline & $\begin{array}{c}\text { Amas Casa } \\
\mathrm{N}=45\end{array}$ & $\begin{array}{c}\text { Trabajadoras } \\
\mathrm{N}=45\end{array}$ \\
& & \\
Edad: & 36,73 & 38,47 \\
Media & 4,01 & 3,38 \\
Sx & & 1,80 \\
\hline $\mathbf{N}^{\mathbf{0}}$ Hijos: & 2,24 & \\
Media & & $9,1 \%$ \\
\hline Estudios: & $13,7 \%$ & $45,4 \%$ \\
Primaria & $52,2 \%$ & $45,5 \%$ \\
Secundaria & $34,1 \%$ & \\
Universitarios & & $47,7 \%$ \\
\hline Nivel de Ingresos: & $55,5 \%$ & $38,6 \%$ \\
Entre 1000 y $3000 €$ & $30,5 \%$ & $13,6 \%$ \\
Entre 3000 y $5000 €$ & $15,5 \%$ & \\
Más de $5000 €$ & & \\
\hline
\end{tabular}

\subsection{Instrumentos}

Estilos educativos parentales. Para la evaluación de los procesos por los cuales un niño aprende las reglas y normas sociales, se ha utilizado la Escala de Estilos Parentales de Irvine, Biglan, Smolowsky y Ary (1999). Se han tomado las dos dimensiones 
principales: permisividad y sobrerreactividad. La escala de permisividad describe las prácticas parentales de bajo control, propias del modelo permisivo de la teoría clásica sobre estilos educativos, mientras que la escala de sobrerreactividad incluye las prácticas de disciplina punitivas y de alto control propias del modelo autoritario. Se ha eliminado el único ítem que comprende la dimensión de supervisión parental ya que no es evolutivamente adecuado para niños menores de 6 años.

Estereotipos de rol familiar. Para la evaluación de los estereotipos de rol familiar se han utilizado 13 ítems de la subescala de Orientación de Rol de la Escala de Satisfacción Marital de Snyder (1989). Esta subescala originalmente consta de 25 ítems en los que se evalúa en qué medida las personas mantienen unas creencias relacionadas con funcionamientos de varón y de la mujer más cercanos a los modelos de familia tradicionales, en los que el varón era el responsable de sostener económicamente a la familia mientras que las tareas de la mujer se centraban en exclusividad en el cuidado y atención de los hijos. Para esta investigación se han elegido expresamente los ítems relacionados con la orientación de rol tradicional descartándose aquellos que se centran en una orientación más igualitaria.

Rol Materno Tradicional. Para valorar el grado de internalización del Rol Materno Tradicional se han elaborado cinco ítems que recogen las cualidades asociadas a una buena madre más mencionadas a lo largo de la literatura por autores como Johnson y Johnson (1977), Solé y Perella (2004) o Moreno (2000). El formato de respuesta de estos ítems es una escala tipo Likert de cuatro puntos cuyas posibilidades de respuesta oscilan entre "en total desacuerdo" (1) y "totalmente de acuerdo" (4).

Estrés parental. El nivel de sobrecarga parental experimentado se evaluó mediante la "Escala de Estrés Parental" (Berry, 2006; Berry y Jones, 1995). Esta escala consta de 18 ítems que representan la consideración de aspectos positivos y agradables (beneficios emocionales, desarrollo y crecimiento personal) y negativos (demandas del medio, coste de oportunidades, restricciones) del desempeño parental. La escala la construyeron los autores con la intención de poder usarse para evaluar el estrés parental tanto en padres como en madres, así como para progenitores con hijos con y sin 
problemas clínicos. Los sujetos evaluados responden sobre su relación con sus hijos en una escala de 1 a 5 que va de "totalmente en desacuerdo" a "totalmente de acuerdo".

Desarrollo socioemocional de los hijos. Para la evaluación de los problemas de conducta se han utilizado dos escalas, la de Retraimiento Social (o aislamiento) y la de Conducta Agresiva del Child Behavior Checklist (CBCL) de Achenbach y Edelbrock (1983). El CBCL es uno de los instrumentos más empleados en clínica infantil, y la fiabilidad y validez de la información que proporciona ha sido demostrada en numerosos trabajos especializados. El instrumento, que distingue entre síndromes de expresión interna y externa, ha sido uno de los más desarrollados y estandarizados, lo que a su vez posibilita el establecimiento de comparaciones interculturales, y son especialmente aptos para la investigación, ya que permiten valorar una amplia gama psicopatológica (Sardinero, Pedreira \& Muñiz, 1997). La escala de Retraimiento Social está compuesta de 9 ítems y la de Conducta Agresiva de 20 ítems.

\section{Resultados}

En primer lugar se llevó a cabo la comparación de las puntuaciones de las mujeres que tienen un trabajo remunerado y las amas de casa en las dimensiones de funcionamiento familiar antes analizadas: rol familiar y materno, estilo educativo y estrés parental. Se han encontrado diferencias significativas en dos aspectos de la ideología de rol (rol materno y rol familiar tradicional) y en dos aspectos de las relaciones familiares (sobrerreactividad y permisividad) aunque la permisividad no llega a ser estadísticamente significativa (Ver Tabla 3). No hay diferencias entre ambas muestras en el nivel de estrés parental. 
Tabla 3. Diferencias de medias entre amas de casa y mujeres con trabajo remunerado

\begin{tabular}{|c|c|c|c|c|c|c|}
\hline & & Media & $\mathbf{t}$ & gl & $\mathbf{P}$ & d \\
\hline \multirow{2}{*}{$\begin{array}{l}\text { Rol materno } \\
\text { tradicional }\end{array}$} & $\mathrm{T}$ & 13,67 & \multirow{2}{*}{$-3,655$} & \multirow{2}{*}{86} & \multirow{2}{*}{$0,0001 * *$} & \multirow{2}{*}{0.78} \\
\hline & A.C.. & 15.95 & & & & \\
\hline \multirow{2}{*}{$\begin{array}{l}\text { Rol familiar } \\
\text { tradicional }\end{array}$} & $\mathrm{T}$. & 12.95 & \multirow{2}{*}{$-4,792$} & \multirow{2}{*}{86} & \multirow{2}{*}{$0,0001 * *$} & \multirow{2}{*}{-1.024} \\
\hline & A.C.. & 17.01 & & & & \\
\hline \multirow[t]{2}{*}{ Sobrerreactividad } & $\mathrm{T}$. & 15.60 & \multirow{2}{*}{$-2,822$} & \multirow{2}{*}{88} & \multirow{2}{*}{$0,006^{*}$} & \multirow{2}{*}{-0.59} \\
\hline & A.C.. & 18.24 & & & & \\
\hline \multirow[t]{2}{*}{ Permisividad } & T. & 11.58 & \multirow{2}{*}{$-1,639$} & \multirow{2}{*}{88} & \multirow{2}{*}{0,105} & \multirow{2}{*}{---} \\
\hline & A.C.. & 13.08 & & & & \\
\hline \multirow[t]{2}{*}{ Estrés parental } & $\mathrm{T}$ & 28,96 & \multirow{2}{*}{$-0,633$} & \multirow{2}{*}{88} & \multirow{2}{*}{0,529} & \multirow{2}{*}{---} \\
\hline & A.C. & 28,14 & & & & \\
\hline
\end{tabular}

Las amas de casa puntúan significativamente más alto que las mujeres con trabajo remunerado en rol materno tradicional y rol familiar tradicional verificándose así la primera hipótesis planteada. Es decir, que con respecto a la ideología de rol, las amas de casa tienden a valorar y desempeñar unos roles familiares propios de un modelo familiar caracterizado porque el marido desempeña el papel de proveedor económico de la familia, es quien tiene el poder de decisión en temas familiares y la mujer es la encargada del cuidado y crianza y de las tareas domésticas, siendo el desarrollo profesional y personal algo secundario. Además tienen una idea de que el ser una buena madre es una capacidad biológica que se tiene de modo instintivo y que se caracteriza por la dedicación en exclusiva a sus hijos/as, dejando a un lado proyectos o necesidades personales.

En lo referente a los estilos educativos, las amas de casa emplean en mayor medida que las mujeres que trabajan un estilo educativo sobrerreactivo. Es decir que tienen una mayor tendencia a utilizar prácticas de disciplina punitivas y de alto control, caracterizadas por reacciones emocionales muy intensas ante la desobediencia de los 
hijos/as (gritar, perder el control de la situación, decir cosas ofensivas...) propias del modelo autoritario de la teoría clásica sobre estilos educativos. Por otro lado, no hay diferencias entre ambos colectivos en relación a la permisividad y, por lo tanto, en esta muestra las madres que tienen un empleo no informan de mayor tolerancia de conductas negativas ni mayor falta de normas y control. Los resultados permiten verificar parcialmente la segunda hipótesis planteada en lo que hace referencia a los estilos educativos permisivos.

Asimismo los datos reflejan la no existencia de diferencias en las vivencias de estrés parental entre las amas de casa y las madres empleadas. El hecho de mantener un trabajo remunerado con las obligaciones y exigencias que de ello se derivan no trae consigo un aumento de insatisfacción y malestar respecto al desempeño del rol parental.

En la comparación de las puntuaciones en las variables de desarrollo socioemocional de los hijos/as tal como informan las amas de casa y las mujeres con trabajo remunerado, no se han encontrado diferencias significativas (ver Tabla 4). Sin embargo, hay que señalar que si bien en aislamiento apenas hay diferencia en las puntuaciones medias, éstas son mucho más marcadas en los comportamientos agresivos y es muy posible que con una muestra mayor hubiesen llegado a ser significativas. Así pues, no hay diferencias en la conducta de los hijos/as percibida por la madre en cuanto a su nivel de agresividad y de retraimiento social según que la madre tenga un trabajo extradoméstico o sea ama de casa. Estos resultados van en la dirección contraria a lo señalado por algunas investigaciones que plantean que el hecho de que los niños/as estén en cuidado no materno durante la primera infancia, estaría relacionado con una mayor frecuencia de conductas agresivas y oposicionistas. Por lo tanto, corroboran la hipótesis planteada por otros autores (Clarke-Stewart, 1989; Hoffman, 2003; Hoffman \& Youngblade, 1999) de que no existen diferencias en estos aspectos de la conducta de los hijos/as por el hecho de que la madre trabaje. 
Tabla 4. Diferencias de medias entre amas de casa y mujeres con trabajo remunerado en el desarrollo socioemocional de sus hijos

\begin{tabular}{|c|c|c|c|c|c|}
\hline & & Media & $\mathbf{t}$ & gl & $\mathbf{p}$ \\
\hline \multirow[t]{2}{*}{ Agresividad } & A.C. & 9.62 & \multirow{2}{*}{-1.922} & \multirow{2}{*}{88} & \multirow{2}{*}{0.058} \\
\hline & $\mathrm{T}$. & 7.60 & & & \\
\hline \multirow[t]{2}{*}{ Aislamiento } & A.C: & 2.27 & \multirow{2}{*}{-1.287} & \multirow{2}{*}{88} & \multirow{2}{*}{0.201} \\
\hline & $\mathrm{T}$. & 1.71 & & & \\
\hline
\end{tabular}

$* * p<0.001 ; * p<0.05$.

A.C: Amas de casa; T: Trabajo remunerado.

\section{Conclusiones}

Este proyecto parte de la realidad social de que cada vez hay mayor número de familias de doble empleo y que, por lo tanto, necesitan conciliar múltiples roles (laborales, conyugales y parentales). Paralelamente a esta realidad social, existen unas creencias compartidas y ampliamente divulgadas que plantean los perjuicios que se derivan para la mujer y, en consecuencia, para el desarrollo de los hijos/as, de simultanear roles, ya que establecen a priori que el desempeño de varios roles tiene que acarrear obligatoriamente desgaste y agotamiento y que el manejo de esa experiencia de estrés pasa obligatoriamente por un desempeño disfuncional y negligente en algunas de las áreas implicadas, en concreto la familiar y el cuidado de los hijos. Desde esta mirada anclada en estereotipos tradicionales propios de unos modelos sociales en los que la mujer era prioritariamente madre, entendiéndose la maternidad como la total disponibilidad para sus hijos pequeños y la consideración de este rol como el rol primario y más importante en la vida de las mujeres, cualquier desviación será interpretable como abandono o negligencia, y por tanto acarreará malestar tanto en la propia mujer como en sus hijos. Sin embargo, son escasos los estudios empíricos que avalan estas tesis o que indagan en las variables que podrían modular los efectos beneficiosos o perjudiciales que acompañan a las circunstancias del desempeño de 
múltiples roles. Nuestros resultados confirman lo establecido por algunas investigaciones que señalan la no existencia de diferencias en relación al desarrollo emocional de los hijos/as por el hecho de que la madre tenga un empleo y que por lo tanto los niños estén en cuidado no materno durante la primera infancia (ClarkeStewart, 1989; Hoffman \& Youngblade, 1999). No se confirma la creencia tan extendida sobre la alta permisividad de las madres que mantienen un trabajo remunerado, ni la imagen tan repetida y presente de una madre con un empleo, insatisfecha y quejosa que vive su situación desde una perspectiva constante de incumplimiento y sobrecarga. Aunque es cierto, y los datos actuales así lo corroboran, que la mujer asume un número mayor de tareas y responsabilidades en el ámbito familiar frente a sus parejas (Dribe \& Stanfords, 2009), sin embargo esto no le hace experimentar sentimientos distintos sobre su experiencia de cuidado y crianza de los hijos que los que experimentan las mujeres que se dedican en exclusividad a atender las demandas familiares.

Finalmente y como era de esperar las madres amas de casa y las que mantienen un trabajo remunerado si que se diferencian en sus creencias sobre los modelos y roles que les corresponderían desempeñar a los varones y mujeres en al ámbito familiar, como a los estereotipos establecidos sobre el desempeño de sus funciones maternas. Las mujeres empleadas han abandonado los modelos tradicionales familiares, que dan primacía al varón como cabeza de familia y proveedor prioritario, en su búsqueda de unos modelos más igualitarios que representen mejor sus experiencias vitales. En esta dinámica se va abandonando la visión de la maternidad como renuncia en aras de una búsqueda de equilibrio, en el que las partes implicadas salgan favorecidas y enriquecidas, entendiendo la maternidad como una experiencia compleja con aspectos positivos y negativos, cuya actuación no está prefijada y que se ejerce en estrecha colaboración con el padre en una relación de similar responsabilidad con los hijos (Moreno, 2000; Hays, 1998). 


\section{BIBLIOGRAFÍA}

- Achenbach, T.M., y Edelbrock, C.S. (1983): Manual for the Child Behavior Checklist and Revised Child Behavior Profile. Burlington, VT: University of Vermont Department of Psychiatry.

- Alberdi, I. (1999): La nueva familia española. Madrid: Taurus.

- Aliaga, C. (2005): Gender gaps in the reconciliation between work and family life. En Statistics in focus: Population and Social Conditions, 4/2005. Disponible en: http://epp.eurostat.ec.europa.eu/cache/ITY_OFFPUB/KS-NK-05-004/EN/KS-NK-05-

\section{4-EN.PDF}

- Arendell, T. (2000): Conceiving and investigating motherhood: the decade's scholarship. En Journal of Marriage \& the Family, 62, pp. 1192-1207.

- Belsky, J. (1990): Developmental risks associated with infant day care: attachment insecurity, noncompliance, and aggression? En S. Chehrazi (ed.) Psychosocial Issues in day care. Washington, DC: American Psychiatry Press.

- Berry, J. (2006): Parental Stress Scale. Disponible en http://www.personal.utulsa.edu/ judy-berry/parent.htm el 2 de Octubre de 2006

- Berry, J.O. y Jones, W.H. (1995): The Parental Stress Scale: Initial psychometric evidence. En Journal of Social and Personal Relationships, 12, pp. 463-472.

- Castles, F.G. (2003): The world turned upside down: below replacement fertility, changing preferences and family-friendly public policy in 21 OECD countries. En Journal of European Social Policy, 13, pp. 209-227.

- Clarke-Stewart, K.A. (1989): Infant day care, maligned or malignant?. En American Psychologist, 44, pp. 266-273.

- Dribe, M., Stanfords, M. (2009): Does parenthood strengthen a traditional household division of labor?. Evidence from Sweden. En Journal of Marriage and Family, 71, pp. $33-45$. 
- Esping-Andersen, G. (1999): Social foundations of postindustrial economies. Oxford, Oxford University Press.

- Gottfried, A.E., Gottfried, A.W. y Bathurst, K. (1995): Maternal and dual-earner employment status and parenting. En M.H. Bornstein (Ed): Handbook of parenting (vol.II). New Jersey: LEA.

- Hays, S. (1998): Las contradicciones culturales de la maternidad. Barcelona: Paidós.

- Hoffman, L.W. (2003): The effects of the mother's employment on the family and the child. Disponible en http://www.parenthood.library.wisc.edu/Hoffman/Hoffman.html. Acceso en 15 de Diciembre de 2003.

- Hoffman,L.W., y Youngblade, L.M. (1999): Mothers at work. Effect on children's well-being. New York: Cambridge University Press.

- Instituto de la Mujer (2005): Conciliación de la vida familiar y la vida laboral: situación actual, necesidades y demandas. Madrid: Instituto de la Mujer.

- Instituto de la Mujer (2008a): Las mujeres en España. Estadísticas de población ocupada. Disponible en: http//www.migualdad.es/mujer/mujeres/cifras/empleo/poblacion_ocupada.htm - Instituto de la Mujer (2008b): Las mujeres en cifras. 1983-2008. Madrid: Instituto de la Mujer. Disponible en http://www.migualdad.es/noticias/pdf/Mujeresencifras.pdf

- Irvine, A.B., Biglan, A., Smolkowski, K., Ary, D. V. (1999): The value of the Parenting Scale for measuring the discipline practices of parents of middle school children. En Journal of Behaviour Research and Therapy, 37, pp. 127-142.

- Johnson, C. L. y Johnson, F. A. (1977): Attitudes toward parenting in dual-career families. En American Journal of Psychiatry, 31, pp. 391-395.

- MacCoby, E., Martin, J.A. (1983): Socialization in the context of the family : parentchild interactions. En E.M. Hetherington y P.H. Mussen (Eds): Handbook of child psychology. Nueva York: Wiley.

- Martínez Díaz, M.P. (2005): El papel de las relaciones familiares en el ajuste familiatrabajo. En M.J. Carrasco y A. García-Mina, El ajuste trabajo-familia desde una perspectiva de género. Madrid: Universidad Pontificia Comillas. 
- Martínez, M.P., Carrasco, M.J. y Aza, G. (2006): Familias de doble empleo: claves diferenciales entre mujeres y varones para un modelo de conciliación de lo personal, familiar y social. Investigación no publicada, Instituto de la Mujer. Disponible en: http://www.migualdad.es/mujer/mujeres/estud_inves/2007/resumen\%20web774.pdf

- Montoro Romero, R. (2000): El papel social y económico de las familias en la configuración del bienestar social. En Dimensiones económicas y sociales de la familia. Madrid: Fundación Argentaria y Visor.

- Moreno, A. (2000): Los debates sobre la maternidad. En C. Fernández-Montraveta, P.

- Monreal, A. Moreno y P. Soto. Las representaciones de la Maternidad. Madrid: Universidad Autónoma de Madrid.

- Musitu, G. (1995): Familia, identidad y valores. En Infancia y Sociedad, 30, pp. 229262.

- NICHD Early Child Care Research Network (2003): Does amount of time spent in child care predict socioemotional adjustment during the transition to kindergarten? En: Child Development, 74, pp.976-1005.

- NICHD Early Child Care Research Network (2005): Child care and child development. New York: The Guilford Press.

- Palacios, J., Hidalgo, M.V. y Moreno, M.C. (2000): Familia y vida cotidiana. En M.J. Rodrigo y J. Palacios (Coords.) Familia y desarrollo humano. Madrid: Alianza.

- Patterson, G.R. (1983): Stress: A change agent for family process. En N. Garmezy \& M. Rutter (Eds.), Stress, coping, and development in children. New York: McGrawHill.

- Prades, J. (2008, 12 de Septiembre): Solos en casa después del cole. En El País pp.32

- Renk,K. Roberts, R. y Roddenberry, A. (2003): Mothers, fathers, gender roles and time parents spend with their children. En Sex Roles, 48, pp. 305-315.

- Sardinero García, E., Pedreira Massa, J.L. y Muñiz, J. (1997): El cuestionario CBCL de Achenbach: Adaptación española y aplicaciones clínico-epidemiológicas. En Clínica y Salud, 8, pp. 447-480. 
- Snyder, D. (1989): Marital Satisfaction Inventory (MSI): (4 ed.): Los Angeles; Western Psychological Services.

- Solé, C. y Perella, S. (2004): "Nuevas" expresiones de la maternidad. Las madres con carreras profesionales “exitosas”. Revista Española de Sociología (RES), 4, pp.67-92. Disponible en http://www.fes-web.org/revista/archivos/res04/03.pdf. Acceso en 7 de marzo de 2006.

- Subirats, M (1993): El trabajo doméstico, nueva frontera para la igualdad. En L. Garrido y E. Gil Calvo (Eds) Estrategias familiares. Madrid: Alianza.

- Tobío C. (2005): Mujeres que trabajan. Dilemas y estrategias, Madrid: Editorial Cátedra.

- Tobío, C. (2002): Conciliación o contradicción: cómo hacen las madres trabajadoras. En Revista Española de Investigaciones sociológicas, 97: pp.155-186.

- VV.AA. (2004): Informe España 2004. Una interpretación de su realidad social. Madrid: Fundación Encuentro.

- Webster-Stratton, C. (1990): Stress: A potential disruptor of parent perceptions and family interactions. En Journal of Clinical Child Psychology, 19, pp. 302-312. 